\title{
Calcineurin inhibitor therapy-associated esophageal polyps 12 years after heart transplantation
}

Drug-induced injury of the gastrointestinal tract is increasingly common but generally under-recognized. Within the esophagus, abnormalities are commonly referred to as "nonspecific pill esophagitis", characterized by erosions, ulcers or occasionally strictures. Alendronate, doxycycline, and potassium chloride have been identified as potential causes [1].

A 63-year-old man who was on long-term calcineurin inhibitor (cyclosporine) therapy due to heart transplantation 12 years earlier, presented with retrosternal pain, odynophagia, and dysphagia. Laboratory tests were unremarkable. The oral cavity was normal on inspection. Endoscopy disclosed multiple glassy polyps measuring up to $5 \mathrm{~mm}$ in diameter within the esophagus ( $\bullet$ Fig. 1 a), of which three were removed by snare polypectomy.

Endoscopic ultrasound showed thickening and splitting of the submucosal layer $(\bullet$ Fig. 1b). Histology revealed massive subepithelial matrix accumulation with edema, fibrosis, and patchy mixed inflammatory infiltrate ( $\bullet$ Fig. 2 ).

Cyclosporine was changed to tacrolimus, and the patient was put on symptomatic therapy with sucralfate. At control endoscopy 6 months later, the number and size of the polyps had markedly decreased and the patient was asymptomatic ( $\bullet$ Fig. 3 ). Gingival overgrowth represents a well recognized side effect of long-term calcineurin inhibitor therapy, occurring in up to $70 \%$ of transplant patients $[2,3]$. The pathogenesis is complex, and several mechanisms have been proposed to explain increased production of matrix and/ or collagen, which is accompanied by reduced activity of matrix metalloproteinases [4]. Less frequently, calcineurin inhibitor therapy-associated inflammatory polyps originate from nongingival tissues of the oral cavity, for example the tongue or the buccal mucosa [4].

The presented case is the first to show calcineurin inhibitor therapy-associated inflammatory polyps within the esophagus. Therapy is mainly symptomatic. If dose reduction is not feasible in a transplant patient, substitution with tacrolimus may be considered which, similar to cyclosporine, can alter fibroblast metabolism, albeit to a lesser extent. In patients suffering

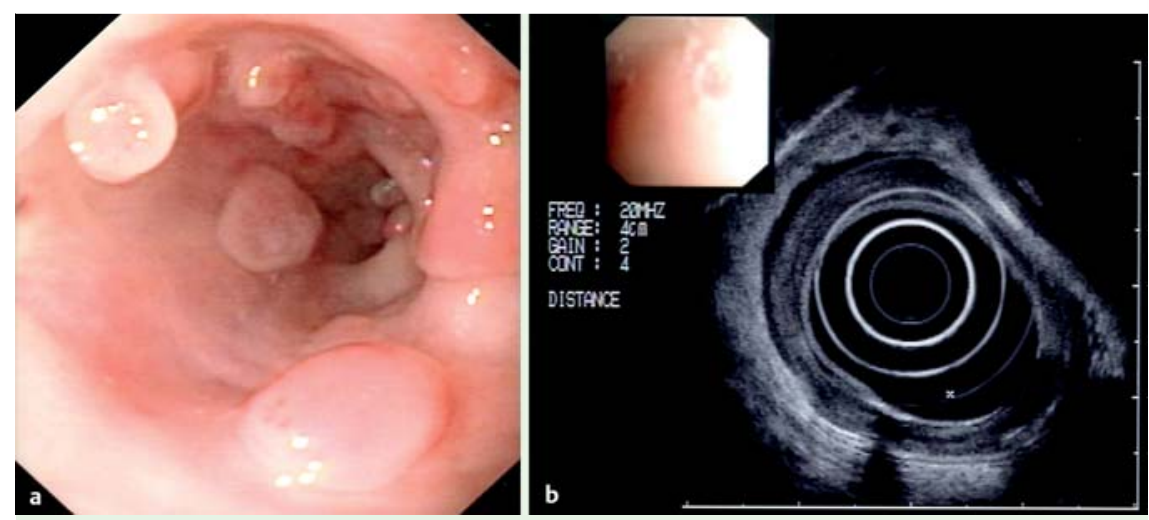

Fig. 1 Calcineurin inhibitor therapy-associated polyps. a Multiple glassy polyps are detected within the esophagus. b Endoscopic ultrasound shows thickening and splitting of the submucosal layer.

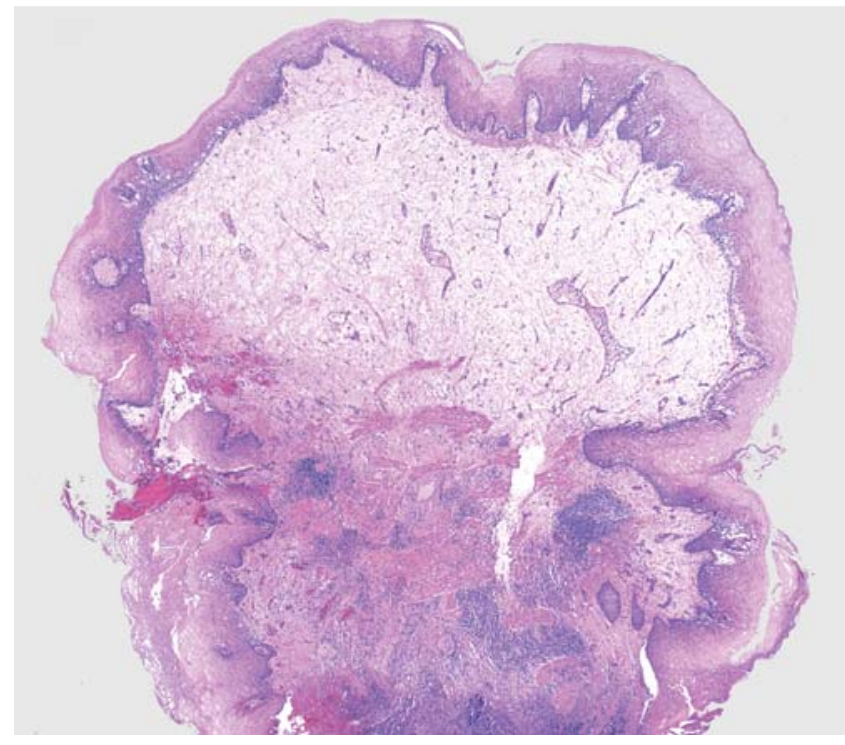

Fig. 2 Histology of calcineurin inhibitor therapy-associated polyps. Hematoxylin and eosin-stained section shows massive subepithelial matrix accumulation with edema, fibrosis, and patchy mixed inflammatory infiltrate.

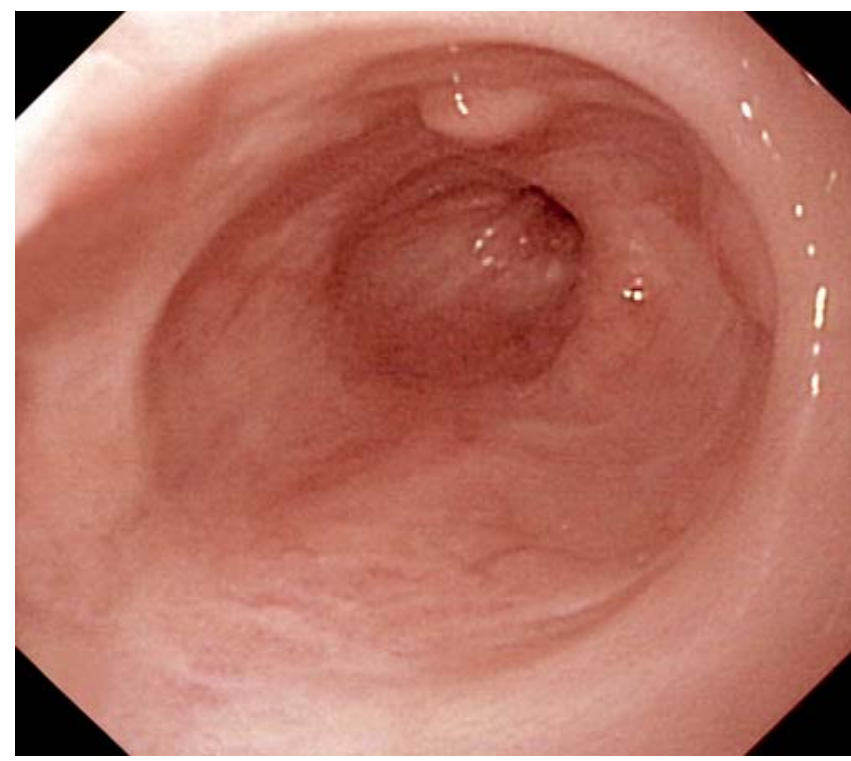

Fig. 3 After 6 months and a change from cyclosporine to tacrolimus, control endoscopy shows marked regression in the number and size of esophageal polyps. 
from autoimmune disease change to an immunosuppressant other than a calcineurin inhibitor is recommended.

Endoscopy_UCTN_Code_CCL_1AB_2AC_3AB

\section{Competing interests: None}

\section{Langner ${ }^{1}$, A. J. Eherer ${ }^{2}$, M. Vieth ${ }^{3}$}

${ }^{1}$ Institute of Pathology, Medical University of Graz, Graz, Austria

2 Department of Internal Medicine, Division of Gastroenterology and Hepatology, Medical University of Graz, Graz, Austria

3 Institute of Pathology, Klinikum Bayreuth, Bayreuth, Germany

\section{References}

1 Parfitt JR, Driman DK. Pathological effects of drugs on the gastrointestinal tract: a review. Hum Pathol 2007; 38: 527-536

2 Wysocki GP, Gretzinger HA, Laupacis A et al. Fibrous hyperplasia of the gingiva: a side effect of cyclosporin A therapy. Oral Surg Oral Med Oral Pathol 1983; 55: 274- 278

3 Daley TD, Wysocki GP, Day C. Clinical and pharmacologic correlations in cyclosporineinduced gingival hyperplasia. Oral Surg Oral Med Oral Pathol 1986; 62: 417-421

4 Al-Mohaya M, Treister N, Al-Khadra $O$ et al. Calcineurin inhibitor-associated oral inflammatory polyps after transplantation. J Oral Pathol Med 2007; 36: 570-574
Bibliography

DOI $10.1055 / \mathrm{s}-0030-1256145$

Endoscopy 2011; 43: E120-E121

(c) Georg Thieme Verlag KG Stuttgart · New York . ISSN 0013-726X

Corresponding author

\section{Langner, MD}

Institute of Pathology

Medical University of Graz

Auenbruggerplatz 25

A-8036 Graz

Austria

Fax: +43-316-38513432

cord.langner@medunigraz.at 